\title{
Vareta de solda impactada em terço médio de face: diagnóstico e tratamento
}

\author{
Welding rod impacted in middle third of gesig: diagnosis and treatment \\ La barra de soldadura impactó en el tercio medio de gesig: diagnosis y tratamiento
}

\begin{abstract}
Resumo
O acidente de trabalho ocorre pelo exercício do trabalho a serviço da empresa, ou pelo exercício do trabalho do segurado especial, provocando lesão corporal ou perturbação funcional, de caráter temporário ou permanente. Esses acidentes representam $1,8 \%$ da causa dos traumas faciais. O objetivo deste trabalho é relatar um caso de ferimento por vareta de solda impactada no terço médio da face após acidente de trabalho, bem como a conduta empregada. Ao exame de imagem, foi possível observar imagem compatível com vareta de solda, transfixado e impactado no terço médio da face. O protocolo efetuado foi assepsia da face, infiltração de anestésico local contendo vasoconstritor, remoção do objeto impactado com porta agulha, controle de hemorragia nasal com a instalado tampão nasal anterior e prescrição de antibioticoterapia. Mesmo não sendo o tipo de lesão mais comum, o trauma maxilofacial pode ocorrer no acidente de trabalho, trazendo consequências emocionais, possibilidade de deformidade irreversível e ao impacto econômico que traz ao sistema de saúde. No caso de lesões transfixantes, o seu tratamento deve priorizar a estabilização do paciente, para sucessivamente ser feita a remoção do objeto. Os acidentes de trabalho são episódios previsíveis e que podem ser evitados, tornando-se necessário para uma melhor abordagem, conhecer sua extensão e gravidade.
\end{abstract}

Palavras-chave: Acidente de trabalho; Ferimentos e lesões; Traumatismos faciais. 


\begin{abstract}
The work accident occurs through the exercise of work at the service of the company, or by the exercise of the work of the special insured, causing bodily injury or functional disturbance, of a temporary or permanent nature. These accidents represent $1.8 \%$ of the cause of facial trauma. The objective of this work is to report a case of injury by welding rod impacted on the middle third of the face after work accident, as well as the conduct employed. At the imaging examination, it was possible to observe an image compatible with a weld rod, transfixed and impacted in the middle third of the face. The protocol performed was asepsis of the face, infiltration of local anesthetic containing vasoconstrictor, removal of the impacted object with needle port, control of nasal hemorrhage with the installed anterior nasal cap and prescription of antibiotic therapy. Even though it is not the most common type of injury, maxillofacial trauma can occur in work accidents, bringing emotional consequences, the possibility of irreversible deformity and the economic impact it brings to the health system. In the case of transfixing lesions, its treatment should prioritize the stabilization of the patient, in case of successive removal of the object. Work accidents are predictable episodes that can be avoided, making it necessary for a better approach, knowing their extent and severity.
\end{abstract} Keywords: Accidents, Occupational; Wounds and injuries; Facial Injuries.

\title{
Resumen
}

El accidente de trabajo se produce por el ejercicio del trabajo al servicio de la empresa, o por el ejercicio del trabajo del asegurado especial, causando lesiones corporales o perturbaciones funcionales, de carácter temporal o permanente. Estos accidentes representan el 1,8\% de la causa del traumatismo facial. El objetivo de este trabajo es reportar un caso de lesión por varilla de soldadura impactada en el tercio medio de la cara tras accidente laboral, así como la conducta empleada. En el examen de imagen, fue posible observar una imagen compatible con una varilla de soldadura, fijada e impactada en el tercio medio de la cara. El protocolo realizado fue asepsia de la cara, infiltración de anestésico local que contenía vasoconstrictor, extracción del objeto impactado con puerto de aguja, control de hemorragia nasal con el capuchón nasal anterior instalado y prescripción de antibioterapia. Aunque no es el tipo de lesión más común, el trauma maxilofacial puede ocurrir en accidentes de trabajo, trayendo consecuencias emocionales, la posibilidad de deformidad irreversible y el impacto económico que trae al sistema de salud. En el caso de lesiones transfixias, su tratamiento debe priorizar la estabilización del paciente, en caso de retirada sucesiva del objeto. Los accidentes de trabajo son episodios predecibles que se pueden evitar, por lo que es necesario un mejor abordaje, conociendo su alcance y gravedad.

Palabras clave: Accidentes de Trabajo; Heridas y Lesiones; Traumatismos Faciales.

\section{Introdução}

De acordo com o artigo 19 da Lei $n^{\circ} 8.213$, de 24 de julho de 1991, "acidente de trabalho é o que ocorre pelo exercício do trabalho a serviço da empresa, ou pelo exercício do trabalho do segurado especial, provocando lesão corporal ou perturbação funcional, de caráter temporário ou permanente". Também é considerado como acidente de trabalho o acidente ocorrido no trajeto entre a residência e o local de trabalho do segurado (De Moura Moreira \& Liberali Magajewski, 2013).

Segundo Wulkan, Parreira Jr e Botter (2005), acidentes de trabalho representam 1,8\% da causa dos traumas faciais, acometendo o sexo masculino em $66 \%$ dos casos e o sexo feminino em $34 \%$ dos casos. Há uma necessidade clara de um maior incentivo por parte das autoridades competentes em ações de prevenção a acidentes, como o uso do capacete, a fim reduzir os traumas, principalmente os faciais (Da Hora Andrade et al., 2021).

A mobilidade da cabeça e a exposição da face em primeiro plano frente aos agentes de trauma foram apontados como alguns dos fatores predisponentes, levando este tipo de trauma a uma incidência de 8 a $9 \%$ quando comparado às outras fraturas do corpo (De Moura Moreira \& Liberali Magajewski, 2013). As estruturas da face e do crânio são capazes de absorver os choques, por causa da presença de pilares resistentes e de cavidades pneumatizadas. Além disso, a face tem uma superfície menor do que o tronco e extremidades. Essas diferenças anatômicas explicam porque lesões penetrantes faciais geralmente resultam em menor grau de lesão ao paciente (Leal et al., 2012).

Essas lesões podem ser causadas por diversos tipos de materiais, como projéteis de arma de fogo, lascas de madeira, objetos metálicos, armas brancas, dentre outros (Morais et al., 2011). O tamanho do objeto e a proximidade de importantes estruturas anatômicas são fatores que devem ser considerados durante a escolha da técnica escolhida para a remoção do corpo estranho; pois o objeto pode atingir importantes estruturas anatômicas causando hemorragia e obstrução das vias aéreas, causando riscos à saúde do paciente (Serra et al., 2016; Lima et al., 2012). 
Entre os exames complementares para deteção e localização de corpos estranhos em face pode-se citar as radiografias planas, tomografia computadorizada e ressonância magnética, sendo as radiografias planas as primeiras a serem utilizadas (Oliveira, et al., 2010; Lima et al., 2014; Cerqueira et al., 2016). A inspeção minuciosa das feridas associadas e a utilização de recursos de imagem são fundamentais para a identificação e localização precisa de corpos estranhos, tendo em vista a possibilidade de ferimentos penetrantes sem sinais clínicos evidentes permanecerem sem diagnóstico (Lima et al., 2014). Vale ressaltar que independentemente da natureza do corpo estranho, todos os pacientes devem ser tratados com antibioticoterapia empírica inicialmente com medicamentos de amplo espectro para prevenir qualquer infecção (Vargas et al., 2010; Casanova et al., 2001).

O objetivo deste trabalho é relatar um caso de ferimento por ferro de solda impactado no terço médio da face após acidente de trabalho, bem como a conduta empregada, e a importância da abordagem precoce por serviços de Cirurgia e Traumatologia Bucomaxilofacial nos serviços de emergência.

\section{Metodologia}

O estudo de caso é um método de pesquisa científica muito utilizado. Ele normalmente consiste em um caso que apresente um fenômeno com alguma característica especial ou diferencial (Pereira et al., 2018). Este artigo é um estudo intervencional descritivo e qualitativo de relato de caso. O paciente o autorizou por meio do Termo de Consentimento Livre e Esclarecido (TCLE), em linguagem acessível que informava sobre o destino das informações recolhidas, benefícios e riscos relacionados à sua participação. Também foi realizada uma revisão da literatura por meio de busca de artigos científicos, relatos de casos, revisões sistemáticas e meta-análises.

\section{Relato de Caso}

Paciente, sexo masculino, 26 anos, melanoderma, compareceu ao serviço de urgência de Cirurgia e Traumatologia Buco Maxilo Facial do Hospital da Restauração, Recife, Pernambuco, Brasil com história de impactação de corpo estranho em face após acidente de trabalho. Paciente sem história de perda de consciência ou episódios eméticos. Ao exame físico apresentava objeto de metal (vareta de solda) de aproximadamente 1,60 mm de diâmetro impactado em região de dorso nasal, transfixando a cavidade nasal e emergindo na região de palato duro (Figura 1).

Figura 1: Paciente no atendimento inicial.

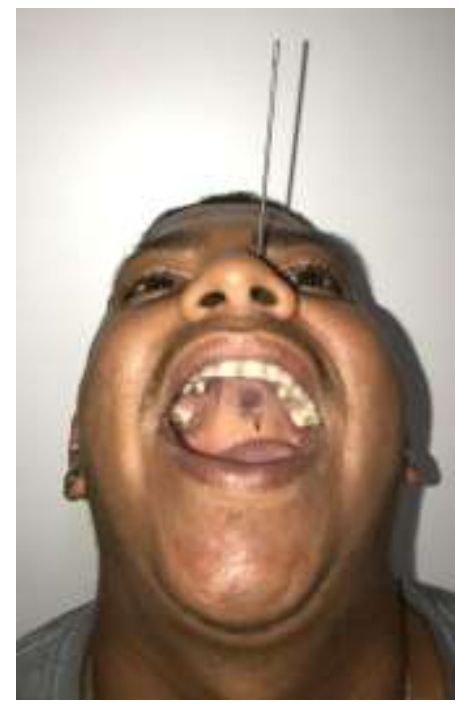

Fonte: Autores. 
No exame de imagem (Radiografia de face em perfil), era possível observar imagem radiopaca, cilíndrica, compatível com vareta de solda, transfixado e impactado no terço médio da face (Figura 2).

Figura 2: Radiografia de face em perfil.

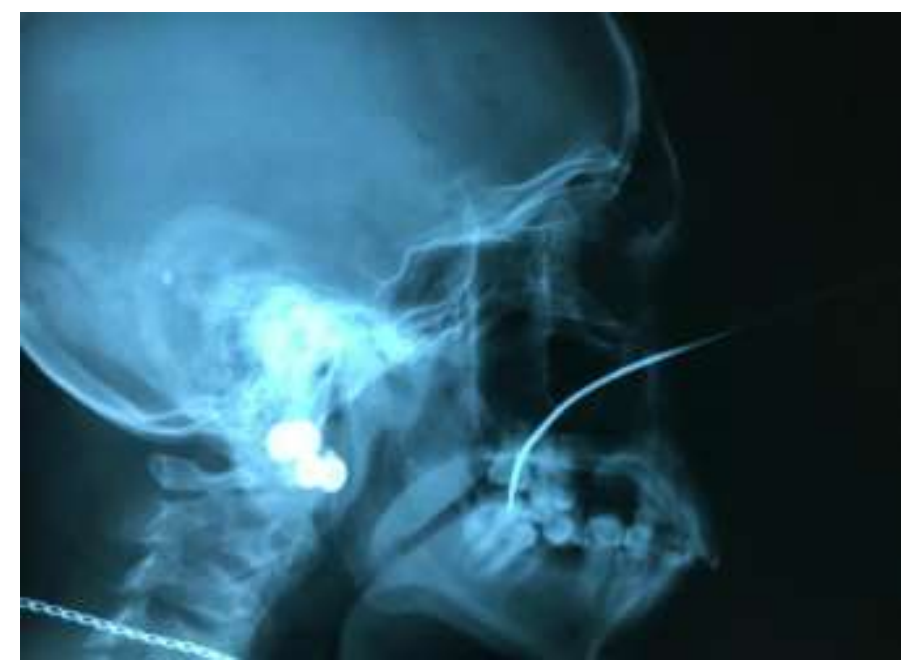

Fonte: Autores.

Dessa forma, foi optado por realizar a remoção do corpo estranho sob anestesia local. Sendo realizada assepsia da face com Clorexidina 2\% degermante, infiltração de anestésico local contendo vasoconstritor (Lidocaína 2\% com Epinefrina 1:100.000) visando anestesiar o nervo infraorbitário bilateralmente, nervo nasopalatino e palatino maior bilateralmente.

Após o bloqueio regional anestésico, foi realizada a remoção do objeto impactado com porta agulha. Após a remoção iniciou-se uma hemorragia intensa em narina esquerda (epistaxe) que foi rapidamente controlado com a instalado tampão nasal anterior do lado esquerdo para promover hemostasia após remoção do corpo estranho, tampão realizado com esponja inserida dentro de um dedo de luva estéril (Figura 3). Foi prescrita antibioticoterapia profilática com Amoxicilina $500 \mathrm{mg}$, via oral, quatro comprimidos por dia, durante sete dias. E o tampão nasal foi removido com 24 horas após o procedimento cirúrgico. Paciente em acompanhamento ambulatorial (follow-up de 6 meses) sem intercorrências e sem alterações estéticas ou funcionais.

Figura 3: Paciente após instalação do tampão nasal anterior.

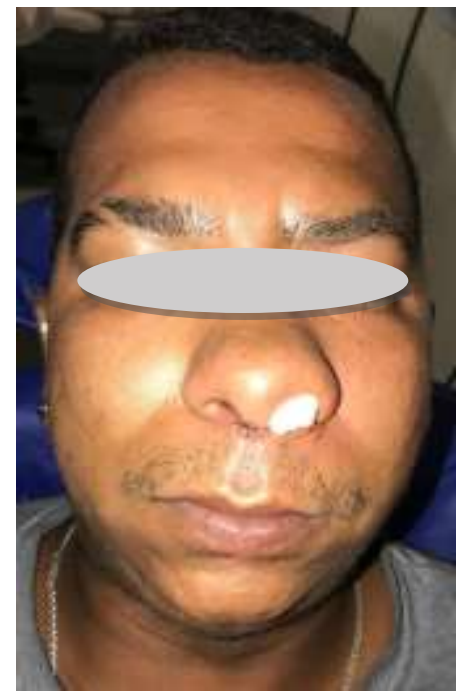

Fonte: Autores. 


\section{Discussão}

Os acidentes de trabalho normalmente acontecem devido a péssimas condições trabalhistas, além da deficiência na utilização dos equipamentos de proteção individual e sobrecarga de horas trabalhadas. Os setores que mais registram acidentes são da indústria de transformação, seguido da construção civil, comércio e agricultura, respectivamente (Conceição et al., 2003).

A maioria dos casos era do sexo masculino (77,8\%), tinha idade acima de 28 anos $(69,7 \%)$, cor negra $(67,7 \%)$ e escolaridade inferior ao ensino médio (72,0\%) (Santana et al., 2009), atingindo mais regiões como cabeça, mãos e pés (Conceição et al., 2003). Esses acidentes podem ser analisados a partir de duas lógicas diferentes: a lógica preventiva e a lógica reparadora (Areosa \& Dwyer, 2010). Neste caso, foi utilizada a lógica reparadora para diminuição de danos ao paciente, visto que não ocorreu a lógica preventiva com o uso dos Equipamentos de Proteção Individual (EPI) durante a manipulação do objeto metálico.

As lesões da cabeça e da face representam metade das mortes traumáticas, e centenas ou milhares de pessoas sobrevivem a essas lesões, muitas vezes com sequelas limitadoras (Ramos et al., 2018). Seu diagnóstico e tratamento obtiveram grande progresso nas últimas décadas, necessitando de abrangência multidisciplinar (Wulkan et al., 2005).

Mesmo não sendo o tipo de lesão mais comum, o trauma maxilofacial pode ocorrer no acidente de trabalho (Wulkan et al., 2005). Quando recorremos à literatura, podemos constatar a sua baixa prevalência nos estudos. Wulkan et al. (2005) encontraram uma prevalência de $1,8 \%$ que sofreram trauma nessa região, enquanto Ramos et al. (2018) encontraram 2,8\%.

Esse trauma possui grande importância por ser um dos mais devastadores devido às consequências emocionais, possibilidade de deformidade irreversível e ao impacto econômico que traz ao sistema de saúde (Wulkan et al., 2005; Ramos et al., 2018). Suas lesões são complexas e podem envolver, dentre outras regiões, ossos, tecidos moles, cérebro, olhos e seios da face (Wulkan et al., 2005). No caso relatado, foram acometidas as regiões de dorso nasal, cavidade nasal e palato duro, livrando algumas estruturas neurovasculares presentes na área.

O trauma maxilofacial acomete em sua maioria homens, entre 20 e 39 anos, e normalmente são feridas contusas e lacerações causadas por violência interpessoal ou acidente motociclístico (Wulkan et al., 2005; Ramos et al., 2018). Os ferimentos penetrantes, como relatados nesse artigo, possuem baixa incidência em face devido ao mecanismo de autodefesa, em que a vítima tende a tentar proteger a face com as mãos, não deixando que o objeto realmente penetre no rosto (Almeida Junior et al., 2010).

Os ferimentos em face podem representar potencial risco à vida, especialmente os que se comunicam com a cavidade nasal ou oral pelo potencial de obstrução de vias aéreas (Bourguignon Filho et al., 2006; Serra et al., 2016). O caso relatado, apesar da comunicação com a cavidade nasal, o paciente apresentava vias aéreas pérvias e respiração mantida, não sendo necessários procedimentos para manutenção das vias aéreas definitivas.

O nariz é uma região bastante irrigada por ser responsável por aquecer o ar antes da entrada no corpo, funcionando como termorregulador. Ele é nutrido pelas artérias etmoidais anterior e posterior, esfenopalatina, palatina maior e ramo septal da artéria labial superior. Além disso, na parte anterior do septo nasal existe plexo arterial anastomótico das cinco artérias e vascularizam o septo, chamado de área de Kiesselbach (Moore \& Dalley, 2011).

Nesse caso clínico, houve epistaxe após a retirada do objeto, isso ocorreu devido a inserção ântero-posterior do objeto, levando ao provável acometimento das artérias etmoidais anterior e esfenopalatina. Segundo Almeida Junior et al. (2010), hemorragias desse tipo podem promover a obstrução das vias aéreas.

Para conter esse sangramento foi utilizada a técnica de tamponamento nasal anterior, que é indicada para sangramentos de grandes volumes. Ela consiste em promover uma compressão direta e difusa sobre toda a mucosa nasal para comprimir os vasos subjacentes (Meirelles, Sá, \& Almeida, 2012). Essa técnica pode ser realizada com uma grande quantidade 
de produtos, no presente caso foi efetuada com dedo de luva estéril preenchidos por espuma também estéril.

Almeida Junior et al. (2010) apresentaram um caso similar ao do presente artigo, onde o paciente apresentou ferimentos corto-contusos em regiões supraorbitária e paranasal, com penetração e impaç̧ão nessa região com ferimento transfixante para região de palato. Esses autores afirmaram que, assim como efetuado no presente caso clínico, o tratamento deve priorizar a estabilização do paciente com avaliação e manutenção das vias aéreas superiores, controle hemodinâmico e avaliação neurológica. Após o atendimento primário, deve ser feita a remoção cuidadosa do objeto pelo mesmo trajeto de entrada. O ferimento deve ser explorado, realizado hemostasia, irrigação copiosa com solução salina e sutura por planos. A remoção desses objetos deve ser realizada de forma a preservar as estruturas dentro do possível, levando em consideração também os fatores estéticos e funcionais envolvidos (Serra et al., 2016).

\section{Conclusão}

Os acidentes de trabalho são episódios previsíveis e que podem ser evitados, tornando-se necessário para uma melhor abordagem, conhecer sua extensão e gravidade. A região maxilofacial é uma das regiões mais acometidas por esse tipo de acidente, sendo o Cirurgião Bucomaxilofacial responsável por reconhecer, diagnosticar e aplicar a conduta adequada com foco na reabilitação funcional e estética do paciente.

\section{Referências}

Almeida Junior, P., Santos, T. S., Kumar, P. N., Filho, P. R. S. M. \& Carvalho, R. W. F. (2010). Ferimento a faca impactada na face (Síndrome de Jael): relato de caso. Revista de Cirurgia e Traumatologia Buco-maxilo-facial, 10 (1), 9-14.

Areosa, J. \& Dwyer, T. (2010) Acidentes de trabalho: uma abordagem sociológica. Configurações. Revista Ciências Sociais, 7, $107-128$.

Bourguignon Filho A. M., Puppin A. A., Pimentel D. P., Jaques P. M., Borges H. O., Lanes S. R., et al. (2006). Unusual penetrating orbit injury. Int J Oral Maxillofac Surg. (35), 92-93.

Casanova, F. H. C., Mello Filho, P. A. A., Nakanami, D. M., \& Manso, P. G. (2001) Corpo estranho orgânico intra-orbitário: avaliação tomográfica e conduta. Arq Bras Oftalmol. 2001, 64: 297-301.

Cerqueira, L. S., Almeida, A. D. S., Rebouças, D. S., Sodré, J. S., \& Marchionni, A. M. T. (2016). Remoção de corpo estranho em seio maxilar: relato de caso. Revista de Cirurgia e Traumatologia Buco-maxilo-facial, 16(2), 44-47.

Conceição, P. S. D. A., Nascimento, I. B. D. O., Oliveira, P. S., \& Cerqueira, M. R. M. (2003). Acidentes de trabalho atendidos em serviço de emergência. Cadernos de Saúde Pública, 19, 111-117.

Da Hora Andrade, M. J., da Silva Limoeiro, A. G., Souza, C. C., Nascimento, W. M., \& Moreira, D. C. (2021). Estudo Epidemiológico de fraturas faciais em uma sub-população brasileira. Research, Society and Development, 10 (5), e27910514937-e27910514937.

De Moura Moreira, L. \& Liberali Magajewski, F. R. (2013). Fraturas faciais em acidentes de trabalho no Brasil-2008 a 2010. Rev. bras. cir. cabeça pescoço.

Leal, J. L. F., Montenegro, J. B., Catunda, I. S., \& Oliveira, D. M. D. (2012). Injúrias penetrantes da face: relato de caso e revisão da literaura. Revista de Cirurgia e Traumatologia Buco-maxilo-facial, 12(2), 43-46.

Lima, E. P. D. A., Bezerra Júnior, A. F. D. A., Leal, J. L. F., \& Cavalcante, A. B. (2012). Presença de corpo estranho orgânico em região frontal: relato de caso. Revista de Cirurgia e Traumatologia Buco-maxilo-facial, 12(2), 59-64.

Lima, E. P. D. A., Lima, T. F. L. D., Leal, J. L. F., Carneiro, S. C. D. A. S., Cavalcante, A. B., \& Sousa Filho, G. C. D. (2014). Presença de corpo estranho no complexo buco-maxilofacial: relato de 2 casos. Revista de Cirurgia e Traumatologia Buco-maxilo-facial, 14(3), 45-52.

Meirelles, R. C., Sá, L. \& Almeida, G. (2012). Abordagem atual das hemorragias nasais, Rev. HUPE, 11 (3), $48-55$

Moore, K.L. \& Dalley, A. F. (2011). Anatomia orientada para a clínica. (6a ed.), Editora Guanabara Koogan S.A.

Morais, H. H. A. D., Silva, A. P. D., Paiva, A. C. S., Medeiros, F. D. C. D. D., \& Araújo, F. A. D. C. (2011). Corpo estranho orgânico em face: relato de caso. Revista de Cirurgia e Traumatologia Buco-maxilo-facial, 11(1), 47-53.

Oliveira, R. S, de Oliveira Costa, R., de Carvalho Neto, L. G., \& Falcão, F. Aplicação da técnica cirúrgica de Caldwell-Luc para remoção de corpo estranho do seio maxilar: relato de caso.

Pereira, A. S., Shitsuka, D. M., Parreira, F. J., \& Shitsuka, R. (2018). Metodologia da pesquisa científica. 
Research, Society and Development, v. 10, n. 13, e220101321152, 2021

(CC BY 4.0) | ISSN 2525-3409 | DOI: http://dx.doi.org/10.33448/rsd-v10i13.21152

Ramos, J. C., Almeida, M. L. D. D., Alencar, Y. C. G. D., de Sousa, L. F., Figueiredo, C. H. M. D. C., \& Almeida, M. S. C. (2018). Estudo epidemiológico do trauma bucomaxilofacial em um hospital de referência da Paraíba. Revista do Colégio Brasileiro de Cirurgiões, 45.

Santana, V. S., Xavier, C., Moura, M. C. P., Oliveira, R., Espírito-Santo, J. S., \& Araújo, G. (2009). Gravidade dos acidentes de trabalho atendidos em serviços de emergência. Revista de Saúde Pública, 43(5), 750-760.

Serra, A. V. P., Sousa, B. C. M. B. D., Figueiredo, L., Azevedo, R., \& Suruagy, W. (2016). Remoção de objeto alojado em terço médio de face: relato de caso. Rev. Odontol. Araçatuba (Online), 60-62.

Vargas, I., Verdugo, F., González, E., \& Pedemonte, C. (2010). Cuerpo extraño de madera en ambas fosas pterigomaxilares: Caso clínico. Revista chilena de cirugía, 62(4), 391-394.

Wulkan, M., Parreira, J. G. \& Botter, D. A. (2005). Epidemiologia do trauma facial. Revista da associação médica brasileira, 51. 